\title{
IV. METHODS OF SAMPLE PREPARATION AND X-RAY DIFFRACTION DATA ANALYSIS, X-RAY MINERALOGY LABORATORY, DEEP SEA DRILLING PROJECT, UNIVERSITY OF CALIFORNIA, RIVERSIDE ${ }^{1}$
}

\author{
H. E. Cook, P. D. Johnson, J. C. Matti, and I. Zemmels, University of California, Riverside
}

\section{INTRODUCTION}

This article describes the methods of sample preparation and X-ray diffraction data analysis of X-ray mineralogy (XM) samples submitted to the DSDP Xray Mineralogy Laboratory at the University of California, Riverside. The sample preparation procedure, the method of diffraction data analysis, and the methods of data handling are designed to efficiently meet the most general needs of geologists in view of the large number and the wide variety of sediment samples submitted for $\mathrm{X}$-ray diffraction analysis.

These methods are shown schematically in Figure 1 and are briefly summarized below. Three preparations of each sample are made for X-ray diffraction analysis: a bulk fraction and decalcified $2-20 \mu$ and $<2 \mu$ fractions. The bulk and $2-20 \mu$ samples are ground to a uniform size. All preparations are treated with trihexylamine acetate to expand the smectites and are X-rayed as random powders.

Raw X-ray diffraction intensities are digitized and recorded on magnetic tape. Mathematically scaled and smoothed diffractograms are generated from the diffraction data on tape. A computer-analysis program is used to make preliminary identification of minerals by diffraction peak position, to measure peak heights, to correct for diffraction peak interferences, and to compute the mineral content by the method of mutual ratios. This computer-generated output is checked manually by geologists with the diffractograms for each sample fraction to confirm the presence of minerals identified by the program and to look for unreported minerals. The original output of the computer program is then updated by computer methods with corrected mineral data; this serves as the data base for computing the percent amorphous and generating mineral tables and histograms.

\section{SAMPLE PREPARATION}

The method of sample preparation is nondestructive (except for grinding) and the sediment fractions can be recovered for further testing or refinement. An exception to this is that calcite, aragonite and, to some extent, dolomite, gypsum, and anhydrite are dissolved in the decalcification process.

Typically, 25-g (10-cc) sediment samples are submitted for X-ray diffraction analysis. Approximately $1 \mathrm{~g}$ is kept as a reference, $5 \mathrm{~g}$ are taken for bulk sample

\footnotetext{
Institute of Geophysics and Planetary Physics, University of California, Riverside, Contribution No. 74-5.
}

preparations, and the remainder is used in preparation of decalcified, fractionated $2-20 \mu$ and $<2 \mu$ samples.

\section{Bulk Samples}

Sediments for bulk sample preparation are disaggregated in Waring blenders with $250-\mathrm{ml}$ hot, distilled water until no lumps of sediment are visible. The samples are centrifuged and the wash-water is decanted. This treatment is sufficient to reduce the seawater salt content below the detection level of X-ray diffraction and also removes some organic residue.

The washed samples are allowed to dry and are disaggregated manually with a mortar and pestle. Coarsegrained samples are reduced to silt size. The samples are then placed in Fisher automatic mortar and pestle grinders and are ground under butanol for 2 hours. The butanol serves to dissipate the heat generated by grinding and also provides a medium for suspending the finer grains. Two hours of grinding under these conditions optimizes the grain sizes of the crystallites for Xray diffraction. After grinding, the butanol is evaporated under heat lamps.

The ground samples are treated with trihexylamine acetate to expand the smectite minerals (synthesis and application of trihexylamine acetate are modified from Rex and Bauer, 1965). The amined samples are permitted to dry for several days to achieve equilibrium with ambient humidity conditions. Trihexylamine acetate has a low vapor pressure and remains in the clay minerals for several weeks. When equilibrated, the amined powders are pressed into sample holders using a custom-built sample press (Rex and Chown, 1960). The crystallites in the mounted powders have a low degree of preferred orientation because of the very fine grain size of the crystallites and because the sample press administers the pressure from the back of the sample.

\section{2-20 $\mu$ and $<2 \mu$ Fractions}

Sediments to be fractionated into $2-20 \mu$ and $\langle 2 \mu$ fractions are first thoroughly disaggregated in Waring blenders and then are decalcified in a sodium-acetatebuffered acetic acid solution $(p \mathrm{H}=4.5)$. Decalcification is performed in an ultrasonic bath which provides heat and agitation to speed the reaction. When decalcification is complete, the reaction products and excess acid are removed by repeated centrifuge washing until the insoluble residue begins to disperse. The residue is then completely dispersed with a $0.05 \%$ sodium hexametaphosphate solution.

These dispersed slurries are passed through a $20 \mu$ sieve with the aid of an ultrasonic probe. The $>20 \mu$ fraction is stored for microscope examination. The slurries with $<20 \mu$ particles are fractionated into $2-20 \mu$ and $<2 \mu$ 


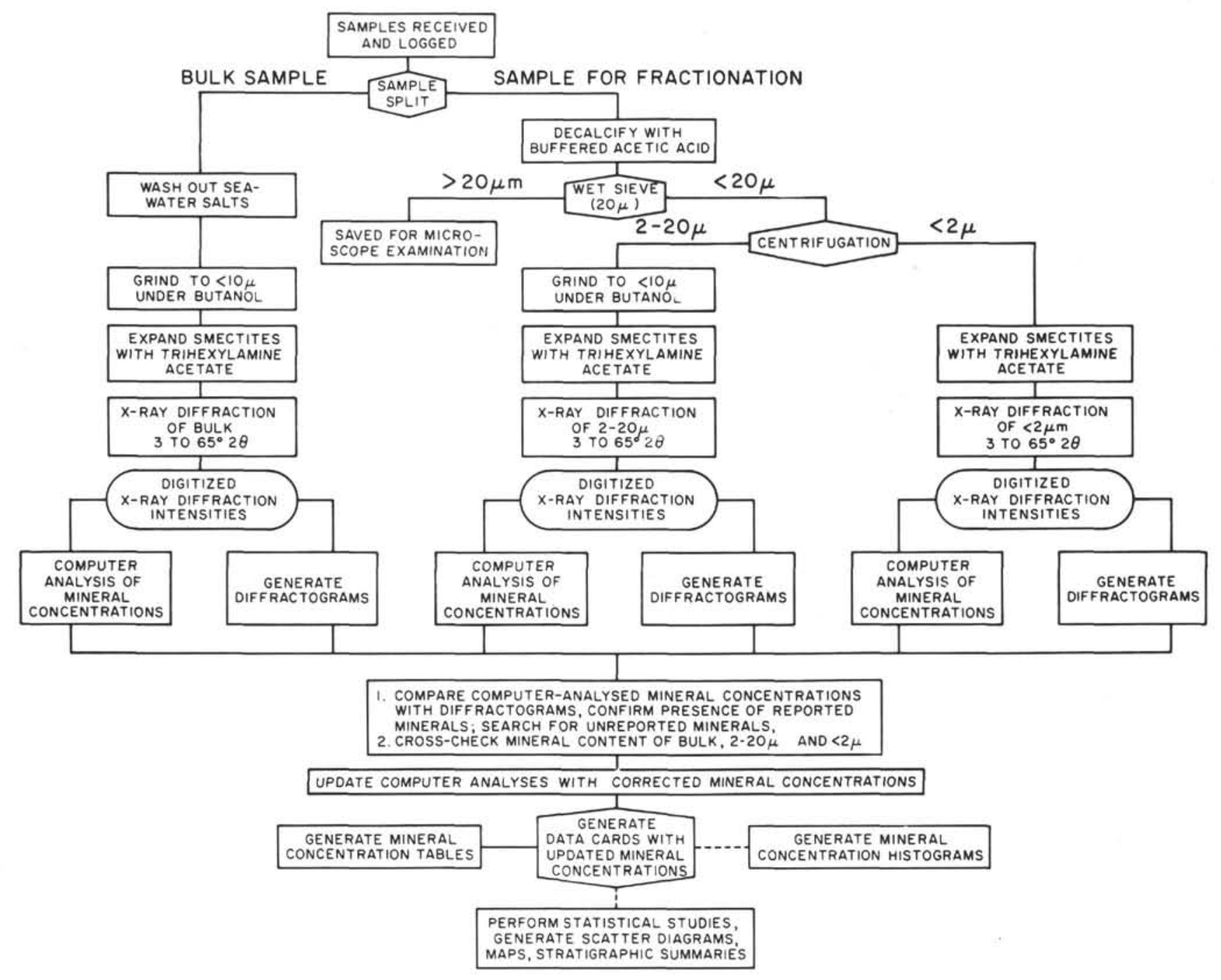

Figure 1. Method of sample preparation and X-ray diffraction analysis.

samples by centrifuging the slurries at a speed and for a time calculated to separate $<2 \mu$ particles. The supernatant liquid containing the $<2 \mu$ particles is collected. Centrifugation of the $<20 \mu$ slurries proceeds until the supernatant liquid is clear. The $<2 \mu$ slurries are flocculated with a few drops of a $10 \% \mathrm{CaCl}_{2}$ solution and are concentrated by centrifugation.

The $2-20 \mu$ samples are ground for 2 hours under butanol in the Fisher grinders whereas $<2 \mu$ samples are not ground. Both of the fractionated samples are treated with trihexylamine acetate to expand the smectite minerals and are mounted as random powders for Xraying in a manner similar to that used for the bulk samples.

\section{Mineral Calibration Standards}

Mineral specimens which are being considered for mineral calibration standards are first subjected to Xray diffraction analysis to determine how well their diffraction pattern resembles the diffraction pattern of minerals found in marine sediments and to determine the mineral impurities present. If the diffraction pattern of the mineral proves it to be acceptable as a calibration standard, any impurities are removed by a variety of methods such as chemical dissolution, heavy liquid separation, or hand picking. The purified standard is mixed with an equal weight of quartz and ground for 2 hours in the Fisher automatic mortar and pestle in order to insure uniformity between samples and standards and to homogenize the standard quartz mixture. Mounting and scanning of the standard quartz mixtures are the same as for the samples.

\section{INSTRUMENTATION}

A Picker powder diffractometer with a graphitecrystal, diffracted-beam monochrometer is used to run the X-ray diffraction scans. A custom, automated sample changer is used instead of the normal sample holder. This gives the capability of loading up to 100 samples at one time. Automated functions of both the diffractometer and the sample changer are controlled by a Digital Equipment Corporation PDP-8 computer 
through a modified Picker FACS-1 interface. The FACS-1 is normally used to control a four-axis, single crystal goniometer.

The PDP-8 computer has 12,000 words of memory. It has two 9-track, $800 \mathrm{bpi}, 25$ ips tape drives which are used for recording and handling of the raw diffraction data in digital form. An 11-inch Houston Instrument Complot digital plotter is used for plotting the diffractogram patterns after they have been smoothed, scaled, and labeled. An 800,000-word disk is used for program and data storage.

The $\mathrm{CuK} \alpha$ lines are used for the scans. Scans are normally $3-65^{\circ} 2 \theta$ at $2^{\circ} / \mathrm{min}$. The patterns are recorded on magnetic tape as a series of 3100 consecutive data points where each data point is the integrated intensity over a $0.02^{\circ} 2 \theta$ interval. The diffraction data can also be traced simultaneously on a strip-chart recorder. One-degree divergence and scatter slits and a 0.01 -inch receiving slit are used. This gives a peak intensity of 30-35 thousand cps on the $26.66^{\circ}$ quartz peak using a novaculite slide. The background in highly crystalline samples varies from a high of $200 \mathrm{cps}$ at $3^{\circ} 2 \theta$ to $50 \mathrm{cps}$ at larger angles. All patterns are plotted on the digital plotter to provide a visual means of verifying and correcting the computerderived analysis.

\section{ANALYTICAL METHOD}

The method of analysis is basically one which is performed by an X-ray diffraction data analyst working with diffractometer tracings. Computer methods in mineral identification, mineral quantification, and data tabulation are employed to perform simple, repetitive functions which are costly in time and are prone to human error. A data analyst checks the results of the computer analysis and manually performs the complicated and/or highly variable mineral identifications and quantifications. The precision of the method is approximately $\pm 1 \%$ by weight for well-crystallized minerals. However, the method is considered to be only semiquantitative because of inevitable differences in the mineralogy of samples and standards.

The method used for the analysis of the X-ray diffractograms is presented below in two sections. The first section lists the 12 steps of the analysis while the second section describes each step. All the steps, except Step 9, are performed by computer. Steps 1 to 8 and Step 11 are performed by a computer-analysis program (MINLOG) and Steps 10 and 12 are performed by a battery of data handling programs. When corrections to the output of the analysis program are made manually, the data analyst duplicates the logic steps used in the computeranalysis program in order to maintain consistency of the data.

\section{Steps in Data Analysis}

1. Smooth the digital pattern.

2. Subtract the background.

3. Determine the location and height of all diffraction peaks.

4. Determine the presence of quartz or calcite using multiple-peak criteria, then correct the location of all peaks using the first mineral identified as a patternalignment standard.

5. Identify minerals present on the basis of a single, diagnostic peak.

6. Subtract interfering secondary peaks of other minerals present from the diagnostic peaks.

7. Multiply all corrected diagnostic peak heights by their intensity factors to convert them into mineral ratios.

8. Adjust the total of all identified minerals to $100 \%$.

9. Manually check the output of the computeranalysis program.

10. Correct the computer output.

11. Estimate the content of amorphous material.

12. Generate mineral-concentration tables and histograms.

\section{Description of Steps in Data Analysis}

1. Smooth the digital pattern: The raw, digitized diffraction data are smoothed mathematically by averaging the intensities of adjacent data points. This is the first step in the computer-analysis program run on the University of California, Riverside, Computing Center's IBM 360-50. (The smoothed data are also used to generate diffractograms of each X-ray sample using the PDP-8 computer and Houston Instrument plotter. The diffractograms are mathematically scaled so that the maximum intensity of any diffractogram equals $10 \mathrm{in}$.) The mathematical method of smoothing is similar to the signal-averaging electronic filters in chart recorders but has the advantage that (a) there is more choice in the weighting of adjacent points, and (b) the filter can be applied to data points in front of, as well as behind, the point being calculated.

A circular filter has been found to be the most effective for smoothing the data. The weighting is derived from the equation

$$
x^{2}+y^{2}=\mathrm{a} \text { constant }
$$

where $x$ is the weighting function, and $y$ is the distance from the point being calculated to the point being used for weighting. Presently a 13-point filter with a constant of 49 is used.

The smoothed intensity value of any point $I^{\prime}$ is given by

$$
I_{i}^{\prime}=\frac{\sum_{j=-6}^{6} x_{j} \cdot I_{i+j}}{\sum_{j=-6}^{6} x_{j}}
$$

where $I i$ is the $i$ unsmoothed intensity point and $j=y$. The 13-point circular filter is approximately the equivalent of a 3-sec time constant.

2. Subtract the background: The background removal routine attempts to separate diffraction peaks from background in a manner similar to that used by a diffraction data analyst. Figure 2 shows a portion of an 

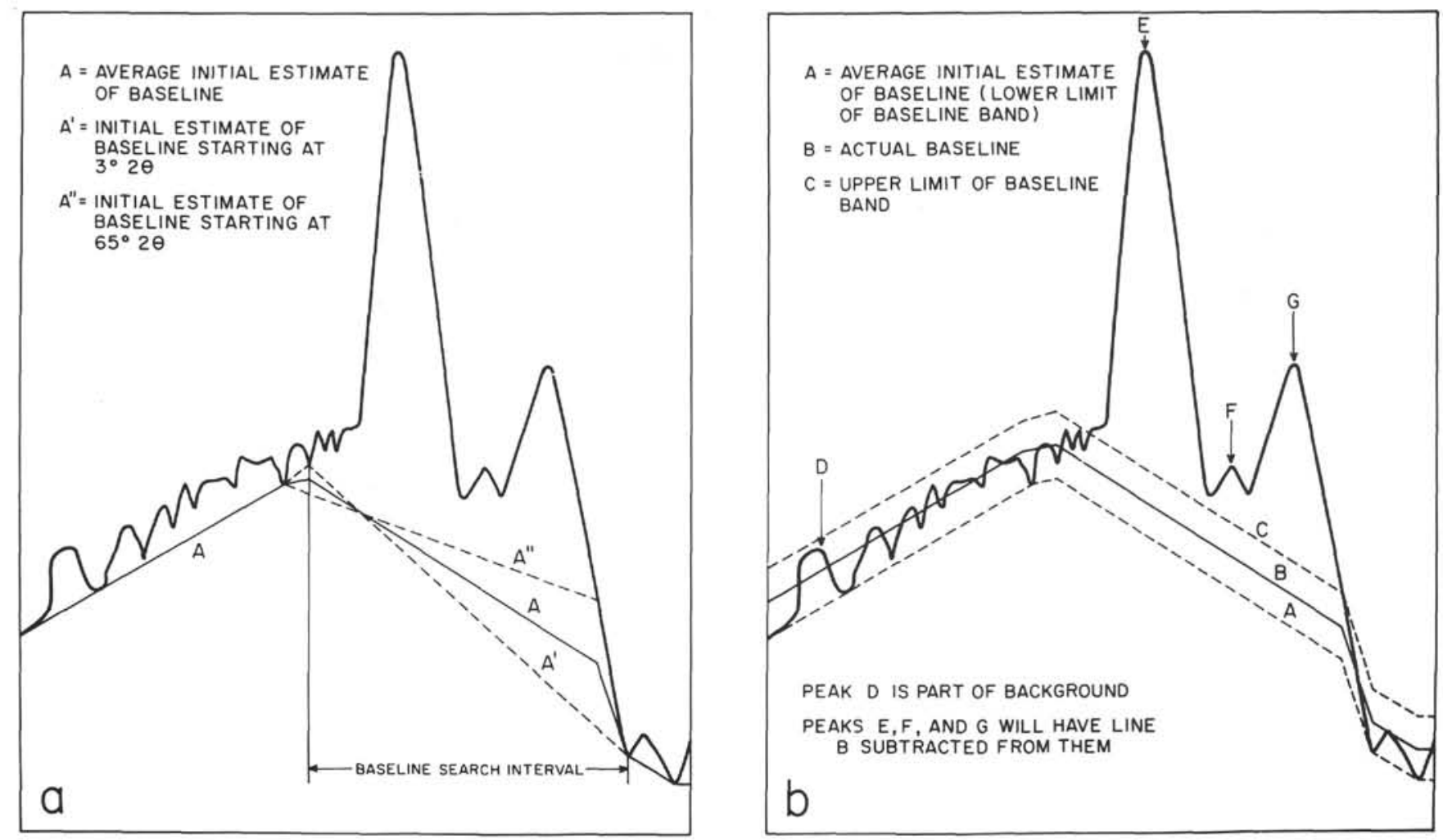

Figure 2. Method of background removal: (a) construction of initial estimate of background, (b) construction of baseline band.

expanded pattern of a clay sample containing a large amount of amorphous silica.

The first estimate of this baseline is made by constructing a series of straight lines from consecutive low points in the pattern. Ideally, these lines have a maximum length which is less than the width of the amorphous hump but greater than the width of any group of overlapping peaks. In practice, the maximum length of these lines, which we call the baseline search interval, is $5^{\circ} 2 \theta$ from $3-11^{\circ} 2 \theta$ and $2^{\circ} 2 \theta$ for the remainder of the pattern. Since these lines are sometimes dependent on the direction from which they are started, the series is started from both ends of the pattern, and the intensity is averaged for each point. In Figure $2 a$, line $A^{\prime}$ is constructed starting from the $3^{\circ}$ end while $\mathrm{A}^{\prime \prime}$ is constructed starting from the $65^{\circ}$ end. Line $A$ is the average of the two lines. Over much of the pattern the lines from the two ends of the pattern coincide.

Since X-rays are given off in a Poisson distribution, the count rate will fluctuate eoen though nothing is changing in the system. The amount of this fluctuation is proportional $t$ the square root of the average count rate. $T$ is fluctuation has two effects: (1) the intensity of the baseline is underestimated since low points are always selected, and (2) some of the intensity fluctuations along the background can appear as small peaks.

The method of background removal assumes that the statistical fluctuation occurs in a band (a range of intensities called the baseline band) above the initial estimate of the background (Figure 2, line A) which is proportional to the square root of the intensity of $\mathrm{A}$ at any point. The band is mathematically constructed above A such that the midpoint of the band is $0.7 \times \sqrt{ } \mathrm{A}$ (line $\mathrm{B}$ ) and the upper limit is $1.4 \times \sqrt{ } \mathrm{A}$ (line $\mathrm{C}$ ). Peaks with intensities less than $\mathrm{C}$, are difficult to resolve from random fluctuation. They are therefore considered as part of the background and are subtracted from the diffraction pattern. Peaks with intensities greater than $\mathrm{C}$ are considered to be real. Their height is computed from line B. For example, in Figure 2, peak D will be considered to be background. Peaks E, F, and G will have the intensity of line B subtracted from them and will be retained for the next step in the computer analysis.

3. Determine the location and height of all diffraction peaks: After the background is removed the residual intensities are tested for the presence of peaks. Currently, a criterion of at least five ascending data points followed by five descending data points is used by the analysis program to define a peak. The actual location and the height of any peak is taken to be the location and height of the most intense data point between the two series of points. Ascending and descending series of points need not be contiguous.

4. Determine the presence of quartz or calcite using multiple-peak criteria, then correct the location of all peaks using the first mineral identified as a patternalignment standard: A number of factors can cause sample peaks to shift as much as $0.3^{\circ} 2 \theta$. These include inaccuracies in positioning of the sample in the sample changer, slight changes in the goniometer alignment, and occasional sample-dimension changes. Although this does not seriously affect manual interpretation of plots, it does make computer identification of minerals much more difficult. Inasmuch as we do not routinely 
use an internal standard, we attempt to use naturally occuring "standards" to make $2 \theta$ corrections. In the bulk fraction, low-magnesium calcite is normally a major constituent, while in all three fractions quartz is frequently a major constituent of the samples.

Quartz is the first mineral sought since it has the most stable crystal lattice. In order to insure that quartz is not missed or misidentified, every peak within $0.8^{\circ} 2 \theta$ of $26.66^{\circ} 2 \theta$ is checked and compared to secondary quartz peaks to see if it is the major quartz peak. The secondary peaks which are checked must be within $0.1^{\circ} 2 \theta$ of the correct distance from the major peak and must have an intensity that is within $30 \%$ of the correct ratios of the major peak. The $20.86^{\circ}$ and $50.18^{\circ}$ peaks are checked, respectively, for intensities of 0.19 and 0.11 of the major quartz peak. The same criteria are applied to calcite. The major calcite peak is $29.44^{\circ}$ and the secondary check peaks are $23.06^{\circ}, 47.56^{\circ}$, and $48.56^{\circ}$ with intensities of $0.086,0.2$, and 0.18 , respectively. Approximately $80 \%$ of the diffractograms can be tested and shifted if needed using the criteria given above.

5. Identify minerals present on the basis of a single, diagnostic peak: The computer identification of the minerals present in a sample is based upon the occurrence of peaks within a narrow range of degrees $2 \theta$ called a window. This window is made as narrow as possible to avoid detecting secondary peaks of other minerals but wide enough to include the diagnostic peak of the target mineral considering variations in the crystal structure, sample positioning, etc.

6. Subtract interfering secondary peaks of other minerals present from the diagnostic peaks: The criteria used in selecting the diagnostic peak for a mineral are: (1) high intensity, (2) low degree of variability in peak position and peak intensity due to crystal-lattice variations, and (3) absence of interfering peaks from other minerals.

The third criterion is difficult to satisfy in sedimentary minerals. In numerous cases the diagnostic peak overlies or is on the shoulder of a secondary peak of another mineral which may occur in the sample. In order to correct for the contribution of an interfering peak to the diagnostic peak height, the proportion of counts appearing in the window of the mineral sought to the number of counts of the diagnostic peak of the interfering mineral is determined. This proportion, called the interference factor, is usually measured from the diffraction pattern of the interfering mineral in pure form. If the interfering mineral is detected in the sample, the peak height in the window of the mineral sought is reduced by an amount determined from multiplying the diagnostic peak height of the interfering mineral by the interference factor.

7. Multiply all corrected diagnostic peak heights by their intensity factor to convert them into mineral ratios: The mineral content is quantified using the method of mutual ratios. Klug and Alexander (1954) have shown that

$$
X_{i}=K_{i} \cdot \frac{I_{i}}{I_{s}}
$$

where

$X_{i}=$ the mineral weight fraction in the sample.

$K_{i}=$ a constant (intensity factor) which is dependent on instrument geometry, the peak intensities of the mineral being analyzed, and the internal diffraction standard.

$I_{i}=$ the intensity of a given peak belonging to the mineral being analyzed.

and

$I_{S}=$ the intensity of a given peak of the internal diffraction standard.

If we form a ratio between minerals $i$ and $j$ we can write

$$
\frac{X_{i}}{X_{j}}=\frac{K_{i} \cdot \frac{I_{i}}{I_{s}}}{K_{j} \cdot \frac{I_{j}}{I_{s}}}=\frac{K_{i} \cdot I_{i}}{K_{j} \cdot I_{j}}
$$

If we assign $K_{j}=1$ and let it always be the same mineral, we can rewrite (2) as

$$
\frac{X_{i}}{X_{j}}=K_{i} \cdot \frac{I_{i}}{I_{j}}
$$

or

$$
X_{i}=K_{i} \cdot I_{i} \cdot \frac{X_{j}}{I_{j}}
$$

If the weight fraction of mineral $j\left(X_{j}\right)$ were known, we could calculate the absolute weight fraction of each mineral present. Since we do not know the weight fraction of mineral $j$ present, we can only calculate the relative fractions for each mineral and say that

$$
X_{i} \propto K_{i} \cdot I_{i}
$$

In practice the $K$ factors are obtained from standards made up of $50 \%$ mineral $i$ and $50 \%$ quartz by weight.

8. Adjust the total of all identified minerals to $100 \%$ : There are two portions of the sample for which we cannot use Equation 7 in determining the relative weight fractions: (1) amorphous material, (2) minerals for which we have no standards. We will call the portion of the sample which is either amorphous or composed of a mineral for which we have no standard the nonquantifiable portion and the remainder the quantifiable portion. If we let $X_{i}$ be the weight fraction of mineral $i$ in the quantifiable portion, we can write

where

$$
X_{i}^{\prime}=a \cdot K_{i} \cdot I_{i}
$$

$$
a=\frac{1}{\sum_{i=1}^{n} K_{i} \cdot I_{i}}
$$


If $A=$ the fraction of the sample which is amorphous and $U=$ the fraction of the sample which cannot be identified or cannot be quantified due to the lack of standard,

$$
X_{i}=(1-A-U) \cdot X_{i}^{\prime}
$$

The output of the computer-analysis program prints the sample identification, the diffuse scatter value of the sample (explained in Step 11), and the peak intensity $\left(I_{i}\right)$ and the relative concentration $\left(X_{i}\right)$ for each identified mineral for manual checking. These data are also stored on magnetic tape for later computer use.

9. Manually check the output of the computer-analysis program: The output of the computer-analysis program is checked against the diffractogram of each X-ray sample by a data analyst. First, the identification of each mineral is confirmed by the presence of secondary peaks of that mineral. Second, the diffractogram is examined for unreported minerals or new minerals. Third, the mineral compositions of the three preparations of one sample (bulk, silt, and clay fractions) are compared for geological consistency among themselves as well as with samples occurring above and below in the stratigraphic column.

If in checking the diffractograms, minerals are found for which interference factors and intensity factors are available, the results of the analysis program are recalculated to include these minerals. Minerals for which factors are not available or which cannot be identified are given an qualitative concentration. A hypothetical intensity factor of 3.0 is assigned for the major peak of any mineral which is to be reported qualitatively. The proportion of the mineral is computed by the method of mutual ratios and is reported according to the following qualitative scale: Trace, $<5 \%$; present, $5-25 \%$; abundant, $25-65 \%$; major, $>65 \%$. Although a certain quantity of the unidentified minerals is implied, their concentration is not included in the concentrations of the identified minerals which are summed to $100 \%$.

10. Correct the computer output: Through a series of computer programs, corrections to the relative concentration percentages as well as minerals which are reported qualitatively are inserted into the original computer output along with descriptive footnotes.

11. Estimate the content of amorphous material: The method by which the content of amorphous material has been estimated since Leg 28 is a modification of the method presented in DSDP Initial Reports, Volume 4, Appendix III. Basically we attempt to measure the amount of amorphous material from the diffuse scatter of a sample. The current method assumes that the diffuse scatter in excess of the sum of the diffuse scatter by the crystalline minerals is a measure of the quantity of amorphous material. It also assumes that the intensity of diffuse scatter per unit weight from the amorphous material is the same as the diffuse scatter from the crystalline components.

The total intensity of an X-ray diffraction pattern can be considered to be a result of five factors: (1) peaks due to Bragg diffraction, (2) diffuse scatter inherent within any crystalline material, (3) diffuse scatter from amorphous material, (4) air scatter, and (5) sample fluorescence, incoherent scatter, extraneous radiation from the instrument, and electronic noise. Air scatter is less than $20 \%$ of the total intensity and remains constant for the goniometer geometry. Sample fluorescence, incoherent scatter, extraneous radiation, and electronic noise are eliminated by the diffracted-beam monochrometer and Pulse Height Analyzer.

The total amount of diffuse scatter, $D$, is calculated by

$$
D=\frac{I_{T}-I_{B}}{I_{T}} \times 100
$$

where $I_{T}$ is the total integrated intensity of diffraction from $3^{\circ}$ to $65^{\circ} 2 \theta$, and $I_{B}$ is the integrated intensity of the Bragg diffraction above the baseline (as computed in Step 2).

A predicted diffuse scatter value, $D_{P}$, is calculated by

$$
D_{P}=0.01 \sum_{i=1}^{n} X_{i}^{\prime} \cdot D_{i}
$$

where $D_{i}$ is the diffuse scattering of the $i^{\text {th }}$ mineral (measured from the standard) and $X_{i}^{\prime}$ percentage of the $i^{\text {th }}$ mineral using corrected data as determined in Step 9. Unknown and qualitatively reported minerals are assumed to have the same diffuse scatter as the quantitatively analyzed portion of the sample. Air scatter is accounted for by the sum of the components.

The percentage of amorphous material present, $A$, is estimated by

$$
A=\frac{D_{s}-D_{p}}{1-D_{p}}
$$

where $D_{S}$ is the diffuse scatter of the sample as determined by Equation 11 .

12. Generate mineral-concentration tables and histograms: A set of cards with sample identification and mineral composition data is punched out by the computer. Cards giving core depths and explanatory comments are inserted to form the complete data decks. These decks are used to generate printed mineralconcentration tables and histograms.

\section{DISCUSSION OF THE METHOD}

The goals of the X-ray Mineralogy Laboratory are to (1) determine the minerals which are present, (2) quantify the ratios between the various minerals, (3) estimate the amount of amorphous material which is present, and (4) determine the individual species of the mineral groups (e.g., solid solution series) which are present, if practical. The list is in the order in which analyses are performed and also is in the order of the priorities. The quality of the mineralogy data will be discussed in this framework. 


\section{Determine the Minerals Which Are Present}

Table 1 is a list of the minerals and mineral groups which are commonly found in sediments and are actively sought by the computer-analysis program and the data analyst. The current windows and intensity factors are also listed. (The lab also has quantitative data for adularia, anorthoclase, anthopyllite, cuprite, grossularite, ilmenite, sphalerite, sylvite, and vermiculite.) Many minerals are identified (and quantified) only on the mineral-group or solid-solution-series level since the mineral species (such as the micas, plagioclases, $\mathrm{K}$ feldspars, and others) have highly similar diffraction patterns and identification of the species becomes impractical using reoutine procedures.

Any analytical method is ultimately limited by its sensitivity; that is, its detection limit and resolution. An experiment was conducted in which gypsum, quartz, dolomite, bytownite, orthoclase, chlorite, apatite, aragonite, and phillipsite were mixed in low concentrations with nannofossil ooze. The samples were randomized and X-rayed by the standard method. The lowest concentration which gave a positive identification of each mineral was deemed to be the detection limit of that mineral. A plot of the intensity factor versus detection limit (in weight percent) gave a straight line which goes to zero and has a slope of 0.12 . Thus the detection limits of quartz and phillipsite are approximately $0.1 \%$ and $2 \%$ by weight, respectively. These detection limits increase linearly with the concentration of diluting amorphous materials and the mass absorption coefficient of the matrix. Interfering peaks also raise the detection limit. Inasmuch as a multiple-peak criterion is used by the analyst to identify most minerals, the actual detection limit will be higher by an amount proportional to the ratio of the diagnostic peak intensity to the secondary peak intensity. In actual pracice we do not report less than $0.3 \%$ quartz and $4 \%$ phillipsite.

The current instrumentation and method of smoothing allows two sharp peaks of equivalent intensity to be resolved if they are more than approximately $0.2^{\circ} 2 \theta$ apart. This permits a resolution of peaks with differences in $d$-spacing of $1 \AA$ at $5^{\circ}, 2 \AA$ at $10^{\circ}, 0.02 \AA$ at $30^{\circ}$, and $0.004 \AA$ at $65^{\circ}$.

TABLE 1

Minerals Actively Sought in Diffraction Data Analysis

\begin{tabular}{|c|c|c|c|}
\hline Mineral & $\begin{array}{c}\text { Window } \\
\left({ }^{\circ} 2 \theta, \mathrm{CuK} \alpha \text { Radiation }\right)\end{array}$ & $\begin{array}{c}\text { Range of } \\
\text { D-Spacings (A) }\end{array}$ & $\begin{array}{r}\text { Intensity } \\
\text { Factor }^{2}\end{array}$ \\
\hline Amphibole & $10.30-10.70$ & $8.59-8.27$ & 2.50 \\
\hline Analcite & $15.60-16.20$ & $5.68-5.47$ & 1.79 \\
\hline Anatase & $25.17-25.47$ & $3.54-3.50$ & 0.73 \\
\hline Anhydrite & $25.30-25.70$ & $3.52-3.46$ & 0.90 \\
\hline Apatite & $31.80-32.15$ & $2.81-2.78$ & 3.10 \\
\hline Aragonite & $45.65-46.00$ & $1.96-1.97$ & 9.30 \\
\hline Augite & $29.70-30.00$ & $3.00-2.98$ & 5.00 \\
\hline Barite & $28.65-29.00$ & $3.11-3.08$ & 3.10 \\
\hline Calcite & $29.25-29.60$ & $3.04-3.01$ & 1.65 \\
\hline Chlorite & $18.50-19.10$ & $4.79-4.64$ & 4.95 \\
\hline Clinoptilolite & $9.70-9.99$ & $9.11-8.84$ & 1.56 \\
\hline Cristobalite & $21.50-22.05$ & $4.13-4.05$ & 9.00 \\
\hline Dolomite & $30.80-31.15$ & $2.90-2.87$ & 1.53 \\
\hline Erionite & $7.50-7.90$ & $11.70-11.20$ & 3.10 \\
\hline Goethite & $36.45-37.05$ & $2.46-2.43$ & 7.00 \\
\hline Gypsum & $11.30-11.80$ & $7.83-7.50$ & 0.40 \\
\hline Halite & $45.30-45.65$ & $2.00-1.99$ & 2.00 \\
\hline Hematite & $33.00-33.40$ & $2.71-2.68$ & 3.33 \\
\hline Kaolinite & $12.20-12.60$ & $7.25-7.02$ & 2.25 \\
\hline K-Feldspar & $27.35-27.79$ & $3.26-3.21$ & 4.30 \\
\hline Magnetite & $35.30-35.70$ & $2.54-2.51$ & 2.10 \\
\hline Mica & $8.70-9.10$ & $10.20-9.72$ & 6.00 \\
\hline Montmorillonite & $4.70-5.20$ & $18.80-17.00$ & 3.00 \\
\hline Palygorskite & $8.20-8.50$ & $10.70-10.40$ & 9.20 \\
\hline Phillipsite & $17.50-18.00$ & $5.06-4.93$ & 17.00 \\
\hline Plagioclase & $27.80-28.15$ & $3.21-3.16$ & 2.80 \\
\hline Pyrite & $56.20-56.45$ & $1.63-1.62$ & 2.30 \\
\hline Rhodochrocite & $31.26-31.50$ & $2.86-2.84$ & 3.45 \\
\hline Quartz & $26.45-26.95$ & $3.37-3.31$ & 1.00 \\
\hline Sepiolite & $7.00-7.40$ & $12.60-11.90$ & 2.00 \\
\hline Siderite & $31.90-32.40$ & $2.80-2.76$ & 1.15 \\
\hline Talc & $9.20-9.55$ & $9.61-9.25$ & 2.56 \\
\hline Tridymite & $20.50-20.75$ & $4.33-4.28$ & 3.00 \\
\hline Gibbsite & $18.00-18.50$ & $4.93-4.79$ & 0.95 \\
\hline
\end{tabular}

${ }^{\mathrm{a}}$ The intensity factors are determined in 1:1 mixtures with quartz by obtaining the ratio of the diagnostic peak intensity of each mineral with the intensity of the diagnostic peak of quartz, which is assigned a value of 1.00. The detection limit in weight percent of the minerals in a siliceous or calcareous matrix can be obtained by multiplying the intensity factor by 0.12 . 
The computer-analysis program is effective in identifying the presence of approximately $90 \%$ of the minerals. The data analyst must identify the remaining mineral phases. This is done by a visual inspection of the diffractograms. In examining the diffractograms, the analyst assigns every major peak (i.e., greater than $10 \%$ ) in the diffraction pattern to a mineral.

The major cause of error in the identification of the common minerals by the computer-analysis program is due to the interference of diffraction peaks. The analyst usually identifies the minerals missed by the program on the basis of secondary peaks. The analyst proceeds to measure the peak height of the diagnostic peak by deconvoluting overlapping peaks and subtracting known interferences. By this method, approximately an additional $9 \%$ are identified and quantified.

The analyst is aided in the rapid recognition of mineral diffraction patterns, even when they occur in small quantities, by the facts that (1) certain minerals occur in natural assemblages (e.g., in volcaniclastic sediments, as characterized by the presence of augite and magnetite, a special effort is made to look for hightemperature feldspars, hematite, ilmenite, analcite, and mordenite); (2) a mineral occurring as a major component in either the bulk, $2-20 \mu$, or $<2 \mu$ fractions commonly occurs as a minor component in another fraction; and (3) a mineral occurring as a significant component at one position in the stratigraphic column may also occur in adjacent samples. Moreover, an experienced analyst develops a keen sense for deviations from "normal" diffraction patterns (produced by the common minerals). The analyst can thereby detect unknown minerals and unusual species of minerals such as highmagnesium calcite, high-temperature feldspars, and mixed-layer clays in concentrations as small as $5 \%$ to $10 \%$.

If there are unassigned peaks in the pattern, an attempt to identify the mineral is made by making an optical examination of the sediment and by checking in the Joint Committee on Powder Diffraction Standards (JCPDS) file. When the peaks cannot be assigned to a known mineral after a reasonable amount of work, the peaks are listed as belonging to an unknown mineral and are published in a footnote.

\section{Quantify the Ratios Between the Various Minerals}

The mineral percentages which are reported are actually ratios of the quantifiable portion of the sample where the total is normalized to $100 \%$. The nonquantifiable portion of the sample consists of the amorphous material, and any mineral group for which we do not at present have an adequate standard (because of insufficient time, nonavailability of pure material, or failure to identify the mineral). Thus a sample which actually contained $5 \%$ quartz, $2 \%$ mica, $43 \%$ an unidentifiable mineral, and $50 \%$ amorphous silica will be reported as $71.8 \%$ quartz, $28.2 \%$ mica, an unidentified mineral as a major component, and an amorphous value of $50 \%$. The mineral and amorphous data are reported with one digit behind the decimal point in order that minerals having low detection limits can be presented. It is not inetnded to imply that degree of accuracy.
The accuracy of quantitative X-ray mineralogy data is principally dependent upon (1) the precision of the method, and (2) the degree of similarity between the minerals in the samples and the calibration standards.

To determine the precision of the quantitative method, a series of 10 samples in which known amounts of calcite, quartz, kaolinite, and dolomite had been intermixed was analyzed. The samples were then run without any attempt to recheck calibration of the mineral standards or alignment of the goniometer. The average deviation of expected versus analyzed concentrations was $3.4 \%$. An examination of the errors revealed that the intensity factors were slightly in error at the time of running. After correcting the factors, the average deviation was $0.4 \%$. Thus the analytical method and method of sample preparation can be expected to give results which are accurate $\pm 0.4 \%$ of the absolute concentration. In practice, replicate samples give concentrations of well-defined minerals which agree within $1 \%$.

The largest source of inaccuracy in the quantitative analysis comes from the differences in crystal structure and the degree of crystallinity between minerals in sediments and mineral calibration standards. For example, a series of high-purity montmorillonites mixed 1:1 with quartz gave intensity factors ranging from 3 to 10 . Diluting impurities in the standards also affect the accuracy.

An effort is made to match the standard with commonly occurring species in the sediment and, if possible, the standards are selected from marine sediments. Nevertheless, the error in the reported values could be as much as $\pm 50 \%$ of the amount present in the case of montmorillonites, $\pm 20 \%$ in the case of micas and chlorites, and $\pm 10 \%$ in solid solution series such as the feldspars and carbonates. Stable forms such as quartz, aragonite, gypsum, barite, pyrite, magnetite, and others will have a reliability approaching $\pm 1 \%$ of the amount present.

In views of the rather high degree of precision of the method, given a homogeneous mineralogy, concentration trends can be regarded as being highly reliable. However, comparisons of mineral concentrations and mineral concentration ratios among lithologic units with different provenances or different diagenetic histories have to take into account the potential differences in the crystal structure of the minerals.

\section{Estimate the Amount of Amorphous Material Which Is Present}

An experiment in which calcite was mixed with $20 \%$, $40 \%, 60 \%$, and $80 \%$ ground glass yielded an average deviation of calculated versus expected concentrations of glass of $2.1 \%$. Thus, in the ideal situation, the amorphous scatter value is an accurate measure of the amorphous material present.

In natural samples, error in the estimate of amorphous material comes from (1) differences in diffuse scatter between the sedimentary minerals and the mineral calibration standards, and (2) difference between the intensity of X-ray scattering between the crystalline phases and the amorphous phases. 
The amorphous scatter value is most accurate at high and low concentrations of amorphous material. It should range between 0 and $100 \%$ but on occasion, slightly negative values of amorphous scatter have been calculated when the diffuse scatter of the identified crystalline minerals in the sample was less than in the standards.

\section{Determine the Individual Species of the Mineral Groups, if It Is Practical}

Normally, distinction between the individual species of a solid solution series or a clay mineral group cannot be made using our methods because further tests are required, or because the sample consists of a mixture of species. When, however, a sample contains a species not commonly found in marine sediments in large concentrations, the species is identified and a footnote is entered in the data tables. Usually the intensity factor of the common form of the mineral is used to quantify the unusual species.

\section{REFERENCES}

Klug, H. P. and Alexander, L. E., 1954. X-ray diffraction procedures for polycrystalline and amorphous materials: New York (John Wiley).

Rex, R. W. and Bauer, W. R., 1965. New amine reagents for X-ray determination of expandable clays in dry samples: Clays and clay minerals, 13th Natl. Conf. Proc., p. 411-418.

Rex, R. W. and Chown, R. G., 1960. Planchet press and accessories for mounting X-ray powder diffraction samples: Am. Mineral., v. 45, p. 1280-1282. 